\title{
ERROR PATTERNS IN DELAYED PITCH COMPARISON AS A FUNCTION OF RELATIONAL CONTEXT ${ }^{1}$
}

\author{
DIANA DEUTSCH ${ }^{2}$ AND PHILIP L. ROLL \\ University of California, San Diego
}

\begin{abstract}
The $S \mathrm{~s}$ made delayed pitch comparisons when the tones to be compared were accompanied by tones of lower pitch. The pitches of these accompanying tones were so chosen that in some conditions the intervals formed by the standard and comparison combinations were identical, and in others they differed. This was true both when the standard and comparison tone pitches differed and also when these were identical. Although $S_{\mathrm{s}}$ were instructed to attend only to the standard and comparison tones, a substantial effect of relational context was manifest. When the standard and comparison tones differed, but were in an equivalent relational context, there resulted an increased tendency for their pitches to be judged as identical. When the standard and comparison tones were identical, but were in different relational contexts, there resulted an increased tendency for their pitches to be judged as different.
\end{abstract}

Previous research has shown that pitch information is retained in a system possessing a high degree of organization and specificity. When two temporally separated tones are compared for pitch, the interpolation of a further tone during the retention interval impairs performance (Wickelgren, 1966). When several tones are interpolated, error rates vary precisely and systematically as a function of the relationships between the test tones and the intervening tones (Deutsch, 1972b). Furthermore, specific sources of memory disruption exhibit partial octave generalization, which shows that this memory system involves both "tone height" and "tone chroma" components (Deutsch, 1973b).

It is clear, however, that the store retaining pitch information must be only a subdivision of the tonal memory system. In listening to music we easily recognize transposed melodies or harmonic sequences as familiar, even though their component pitches have altered. Indeed, providing that sufficient time or interference occurs between the initial presentation of a musical sequence and its repetition in transposed form, we may even become unaware of a

1 This work was supported by U. S. Public Health Service Grant MH-21001-03.

${ }^{2}$ Requests for reprints should be sent to Diana Deutsch, Department of Psychology, University of California, San Diego, P. O. Box 109, La Jolla, California 92037. change of key (Attneave \& Olson, 1971; Deutsch, 1969). One must conclude that, compared with absolute pitch values, relational information is held very securely in memory. The question therefore arises as to how these two types of information interact in memory.

The simultaneous presentation of two tones results in the perception of a musical interval; and pairs of tones form intervals of the same apparent size when their waveform frequencies are related by the same ratio. Since the musical scale is logarithmic, pairs of tones appear to stand in the same relationship when the members of each pair are separated by the same distance along this scale (i.e., by the same number of semitones).

In the present study, $S$ s were required to make delayed pitch comparison judgments when the tones to be compared were accompanied simultaneously by tones of lower pitch. The accompanying tones were presented to a different ear and $S \mathrm{~s}$ were instructed to ignore them. The pitches of these accompanying tones were so chosen that in some conditions the intervals formed by the components of standard and comparison combinations were identical, and in other conditions they differed. Further, these different patterns of relationship were present both in conditions where standard and comparison tone pitches were identical, and also where they differed. This general 


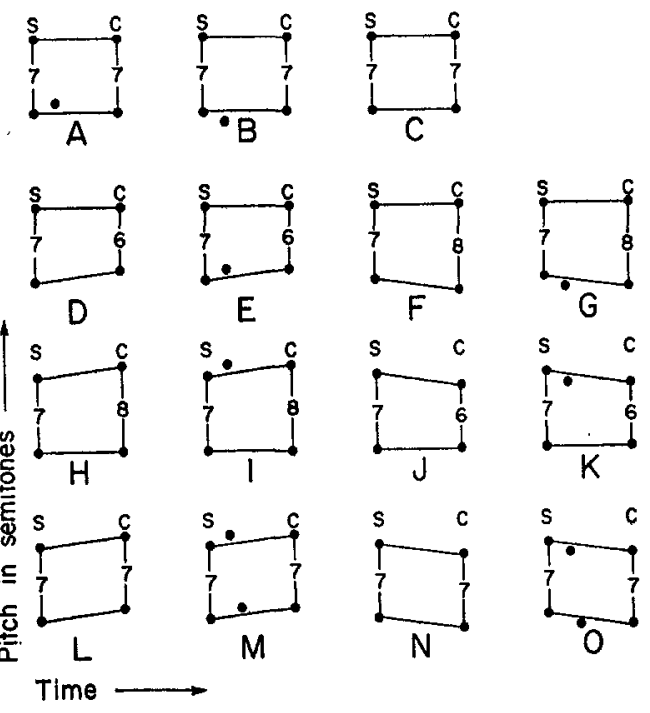

Figure 1. Diagrammatic representation of the conditions of Experiment I. ( $\mathrm{S}=$ standard combination, $\mathrm{C}=$ comparison combination. Vertical distance between two circles represents interval size in semitones. Circles placed intermediate between the standard and comparison combinations represent critical intervening tones and their pitches relative to those of the standard and comparison combinations.)

design enabled an overall determination of the effect of concordant and conflicting relational information on pitch comparison judgments. It was hypothesized that a substantial influence of relational context would be manifest. Further, the standard and comparison combinations were always separated by a retention interval during which other tones were interpolated. Tones bearing specific relationships to the components of the standard or comparison combinations were systematically included in the intervening sequence, in order to provide a better understanding of the interactions obtained.

\section{Method}

\section{EXPERIMENT I}

Procedure. The $S$ s listened to a standard tone, which was followed by a sequence of six interpolated tones, and then, after a pause, by a comparison tone. The standard and comparison tones were always accompanied by tones of lower pitch. The Ss were instructed to listen to the upper tone of the standard combination and ignore the lower tone, to ignore the six intervening tones, and then to judge whether the upper tone of the comparison combination was the same or different in pitch from the upper tone of the standard combination. They then indicated their judgments by writing " $\mathrm{S}$ " (same) or "D" (different) on paper. Before embarking on the experimental session, $S$ s were played several examples of these sequences, and care was taken to insure that they understood the task.

Temporal parameters. In each sequence, all tones were $300 \mathrm{msec}$. in duration. The time interval between the standard combination and the first intervening tone was $500 \mathrm{msec}$, and the intervening tones were also spaced $500 \mathrm{msec}$. apart. A 2-sec. pause was incorporated between the last intervening tone and the comparison combination. Sequences were separated by 10 -sec. pauses; and an extra 2 -min. pause was incorporated at the end of every 12 sequences.

Conditions. In all conditions, the standard tone was separated from its accompanying tone by an interval of seven semitones (a musical fifth). However, the relationship between the comparison tone and its partner varied depending on the experimental condition.

There were seven conditions in which the standard and comparison tones were identical. These are shown in diagram form in Figure 1. In Conditions $\mathrm{A}, \mathrm{B}$, and $\mathrm{C}$, the tones accompanying the standard and comparison tones were also identical in pitch, so the intervals formed by the standard and comparison combinations were also identical in size. In Condition $\mathrm{A}$, a tone that was a semitone higher than the tone accompanying the standard (and comparison) tone was included in the second serial position of the intervening sequence. In Condition $\mathrm{B}$, a tone that was a semitone lower was so included. In Condition $\mathrm{C}$, no tone a semitone removed from the standard (and comparison) tone was included in the intervening sequence. In Conditions $\mathrm{D}, \mathrm{E}$, $F$, and $G$, the tone accompanying the comparison tone was a semitone removed from the tone accompanying the standard tone, so the intervals formed by the standard and comparison combinations also differed in size by a semitone. In Conditions $\mathrm{D}$ and $\mathrm{E}$, the tone accompanying the comparison tone was a semitone higher than the tone accompanying the standard tone; in Conditions $\mathrm{F}$ and $\mathrm{G}$ it was a semitone lower. Further, in Conditions $E$ and $G$, a tone that was identical in pitch to the tone accompanying the comparison tone was included in the second serial position of the intervening sequence. In Conditions $\mathrm{D}$ and $\mathrm{F}$, no such tone was included.

There were eight conditions in which the standard and comparison tones differed in pitch by a semitone. These are also shown in diagram form in Figure 1. In Conditions $\mathrm{H}, \mathrm{I}, \mathrm{J}$, and $\mathrm{K}$, the pitches of the accompanying tones remained the same, so that the intervals formed by the standard and comparison combinations also differed in size by a semitone. In Conditions $\mathrm{H}$ and $\mathrm{I}$, the comparison tone was a semitone higher in pitch than the standard tone; and in Conditions $\mathrm{J}$ and $\mathrm{K}$, it was a semitone lower. Further, in Conditions $I$ and $K$, a tone that 
was identical in pitch to the comparison tone was placed in the second serial position of the intervening sequence. In Conditions $L, M, N$, and $O$, the tones accompanying the standard and comparison tones differed in pitch by a semitone. This difference was always in the same direction as the difference between the standard and comparison tones, so the intervals formed by the standard and comparison combinations were identical in size. In Conditions $\mathrm{L}$ and $\mathrm{M}$, the comparison tone was a semitone higher than the standard tone, and in Conditions $\mathrm{N}$ and $\mathrm{O}$, it was a semitone lower. Further, in Conditions $M$ and $\mathrm{O}$, a tone of the same pitch as the comparison tone was included in the second serial position of the intervening sequence, and a tone of the same pitch as the tone accompanying the comparison tone was included in the third serial position.

Each condition employed one example of each of the standard combinations (see below), except for Condition C, which employed two. (The reason for doubling the number of sequences in Condition $C$ was to equalize the number of sequences where the standard and comparison tones were the same in pitch compared to different; where the lower tone shifted compared to where it remained the same; and where a critical tone was included compared to not included.) The tape therefore consisted of 96 sequences. The $S$ s listened to the entire tape on 2 separate days and the results were averaged. Each $S$ therefore made 12 judgments for each condition, except for Condition $\mathrm{C}$, where he made 24 ,

Tonal stimuli. These were taken from an equal tempered scale (international pitch; $A=435$ ) and ranged from $A$ \# below middle $C$ to the $E$ over an octave above. The following frequencies (in hertz) were employed: $\mathrm{A} \#=230, \mathrm{~B}=244, \mathrm{C}=259, \mathrm{C}$ $=274, \mathrm{D}=290, \mathrm{D} \#=308, \mathrm{E}=326, \mathrm{~F}=345$, $F \#=366, G=388, G \#=411, A=435, A \#=461$, $\mathrm{B}=488, \mathrm{C}=517, \mathrm{C} \#=548, \mathrm{D}=581, \mathrm{D} \#=516$, and $E=652$. The following standard combinations were employed: $F=345, A \#=230 ; G=388$, $\mathrm{C}=259 ; \mathrm{A}=435, \mathrm{D}=290 ; \mathrm{B}=488, \mathrm{E}=326$; $C \#=548, F \#=366 ;$ and $D \#=615, G \#=411$. The interpolated tones, except as specified by the experimental condition, were chosen randomly from the frequencies listed above, with the following restrictions. No sequence included repeated tones, or tones separated by octaves. No intervening sequence included tones that were the same in pitch as either the standard tone or the tone accompanying the standard tone, or that was separated from either by a semitone; and no sequence contained tones that were exactly an octave removed from any of these.

Subjects. Sixteen undergraduates at the University of California at San Diego served as $S$ s for the experiment. They were selected on the basis of obtaining a score of at least $90 \%$ correct on a small tape containing similar sequences, but where the standard and comparison tones were not accompanied by tones of lower pitch. The Ss were paid for their services.

A pparatus. Tones were generated by two Wavetek oscillators controlled by a PDP 8 computer, and the output was recorded on high fidelity tape. The tape was played to $S$ s on a high quality tape recorder, the output of which was passed through a frequency balance control (Advent Corporation) and a Fisher Stereo Master Control Amplifier, with the controls adjusted so that the tones were equal in apparent loudness. The output was played to $S$ s through earphones. The two components of each standard and comparison combination were fed to separate ears, and the intervening tones were fed simultaneously to both ears.

\section{Results}

The results were analyzed separately for sequences where the standard and comparison tones were identical in pitch, and for sequences where they differed.

Standard and comparison tones identical. The error rates for Conditions A, B, C, D, $\mathrm{E}, \mathrm{F}$, and $\mathrm{G}$ were, respectively, 5.7, 9.4, 7.6, 31.3, 31.8, 40.6, and 11.5. The results were analyzed in a $2 \times 2 \times 2$ repeatedmeasures analysis of variance, with the second and third factor nested within the first. The three factors tested were the effect of a lower tone shift (Conditions $A$, B, C vs. D, E, F, G); the effect of direction of lower tone shift (Conditions $D, E$ vs. $F, G)$; and the effects of the critical interpolated tone (Condition $C$ vs. $A, B$, and Conditions $D, F$ vs. E, G).

The effect of a lower tone shift was found to be highly significant, $F(1,15)=$ $43.1, p<.01$. Indeed, a substantial increment in errors occurred when the standard and comparison tones were placed in different relational contexts (Table 1). The effect of the critical interpolated tone was highly significant given a lower tone shift, $F(1,15)=16.7, p<.01$. However, this effect only occurred when the lower tone shifted downward but not upward; and, as expected, the interaction between the effect of the interpolated tone and the direction of lower tone shift was highly significant, $F(1,15)=12.29, p<.01$. There was no effect of the interpolated tone where the lower tone remained the same, $F<1$, and there was no overall effect of direction of lower tone shift, $F(1,30)=2.92, p>.05$.

Standard and comparison tones different. The error rates for Conditions $\mathrm{H}, \mathrm{I}, \mathrm{J}, \mathrm{K}$, $\mathrm{L}, \mathrm{M}, \mathrm{N}$, and $\mathrm{O}$ were respectively, 5.2, 34.4, $3.7,27.1,7.8,53.1,17.2$, and 43.8 . The 
TABLE 1

Effect of Changing Relational Context in EXPERIMENT I: STANDARD AND Comparison Tones Identical in Pitch

\begin{tabular}{c|c|c|c}
\hline Tones and intervals & \multicolumn{3}{|c|}{ Condition } \\
\cline { 2 - 4 } & $\mathrm{A}, \mathrm{B}, \mathrm{C}$ & $\mathrm{D}, \mathrm{E}$ & $\mathrm{F}, \mathrm{G}$ \\
\hline $\begin{array}{c}\text { Lower tone of comparison } \\
\text { combination compared to } \\
\text { that of standard } \\
\text { combination }\end{array}$ & Same & $\begin{array}{c}\text { Semitone } \\
\text { higher }\end{array}$ & $\begin{array}{c}\text { Semitone } \\
\text { lower }\end{array}$ \\
$\begin{array}{c}\text { Interval formed by } \\
\text { comparison combination } \\
\text { compared to that formed } \\
\text { by standard combination }\end{array}$ & Same & $\begin{array}{c}\text { Semitone } \\
\text { smaller }\end{array}$ & $\begin{array}{c}\text { Semitone } \\
\text { larger }\end{array}$ \\
$\% \%$ errors & 7.6 & 31.5 & 26.0 \\
\hline
\end{tabular}

results were analyzed in a $2 \times 2 \times 2$ repeated-measures analysis of variance with the second and third factors nested within the first. The three factors tested were the effect of a lower tone shift (Conditions $\mathrm{H}, \mathrm{I}, \mathrm{J}, \mathrm{K}$ vs. $\mathrm{L}, \mathrm{M}, \mathrm{N}, \mathrm{O}$ ), the effect of whether the comparison tone was lower or higher than the standard tone (Conditions $\mathrm{H}, \mathrm{I}$ vs. J, K, and Conditions $\mathrm{L}, \mathrm{M}$ vs. $\mathrm{N}, \mathrm{O}$ ), and the effects of the critical interpolated tones (Conditions $\mathrm{H}, \mathrm{J}$ vs. I, K, and Conditions $\mathrm{L}, \mathrm{N}$ vs. $\mathrm{M}, \mathrm{O}$ ).

The effect of a lower tone shift was found to be highly significant, $F(1,15)=$ 53.6, $p<.01$. As is shown on Table 2, a substantial increase in errors occurred when the lower tones of the standard and comparison combinations shifted in parallel with the higher tones, so that the relationship within the combinations was preserved. In a separate test involving only sequences where no critical tones were interpolated (Conditions $\mathrm{H}, \mathrm{J}$ vs. $\mathrm{L}, \mathrm{N}$ ), this effect was still found to be highly significant, $F(1,15)=9.91, p<.01$. The overall significance was not, therefore, due to the effect in sequences containing critical interpolated tones alone. The effect of the critical interpolated tones was also highly significant, both when the lower tones shifted, $F(1,30)=95.4, p<.01$, and also when they remained the same, $F(1,30)$ $=50.1, p<.01$. There were no significant effects depending on whether the comparison tone was lower or higher than the standard tone, neither when the lower tone shifted, $F<1$, nor when it remained the same, $F(1,30)=1.78, p>.05$.

\section{Discussion}

It can be seen that errors in pitch comparison increase substantially in the presence of conflicting relational information. Thus, in sequences where the standard and comparison tones are identical, errors increase when their accompanying tones differ so that the standard and comparison combinations form different intervals (Table 1). Similarly, in sequences where the standard and comparison tones differ, errors increase when the lower tones shift in a parallel direction, so that the standard and comparison combinations form identical intervals (Table 2). It therefore appears that memory for the relationship within a tonal combination is so powerful that it substantially interferes with memory for one of its components, even after only a few seconds have elapsed.

Other possible interpretations must, however, be considered. One is that $S$ s were simply judging the wrong tone. This argument cannot apply to sequences where the standard and comparison tones differ, since here there are increased reports of same when the lower tones also differ compared with when they remain the same. However, in sequences where the standard and comparison tones are identical, a difference in the size of the interval formed by the standard and comparison combinations must of necessity be produced by a difference in the pitches of the lower tones. The question therefore arises as to what extent errors here were due to the recognition of a different tone in the comparison combination, rather than to the recognition of a different interval.

This question was explored along various lines. First, it was reasoned that if $S_{S}$ were indeed judging the wrong tone, then their judgments of whether the comparison tone was higher or lower than the standard tone should depend on the direction in which the lower tone pitches shifted. Accordingly, the tape was again presented to these $S$ s, but they were required this time to judge whether the comparison tone was the same as, higher than, or lower than the standard tone. No correlation was found between the direction of apparent pitch difference between the standard and comparison tones when they were really identical, and the direction in which their accompanying tones differed. Yet $S$ s showed only $2 \%$ errors in such judgments of direction when the standard and comparison tones did in fact differ, so this 
TABLE 2

Effect of Changing Relational Context in Experiment I: Standard and Comíparison Tones Different in Pitch

\begin{tabular}{|c|c|c|c|c|}
\hline \multirow{2}{*}{ Tones and intervals } & \multicolumn{4}{|c|}{ Condition } \\
\hline & $\mathrm{H}, \mathrm{I}$ & $\mathrm{J}, \mathrm{K}$ & $\mathrm{L}, \mathrm{M}$ & $\mathrm{N}, \mathrm{O}$ \\
\hline $\begin{array}{l}\text { Comparison tone compared to standard tone } \\
\text { Lower tone of comparison combination compared } \\
\text { to that of standard combination } \\
\text { Interval formed by comparison combination com- } \\
\text { pared to that formed by standard combination } \\
\text { \% errors }\end{array}$ & $\begin{array}{l}\text { Semitone } \\
\text { higher } \\
\text { Same } \\
\text { Semitone } \\
\quad \text { larger } \\
19.8\end{array}$ & $\begin{array}{l}\text { Semitone } \\
\text { lower } \\
\text { Same } \\
\text { Semitone } \\
\text { smaller } \\
\\
15.4\end{array}$ & $\begin{array}{l}\begin{array}{c}\text { Semitone } \\
\text { higher } \\
\text { Semitone } \\
\text { higher }\end{array} \\
\text { Same } \\
\quad 30.5\end{array}$ & $\begin{array}{l}\begin{array}{c}\text { Semitone } \\
\text { lower } \\
\text { Semitone } \\
\text { lower }\end{array} \\
\text { Same } \\
\quad 30.5\end{array}$ \\
\hline
\end{tabular}

lack of correlation was not due to an inability to use the "higher" and "lower" categories.

Two further attempts were made to confirm the hypothesis that $S$ s were sometimes judging the wrong tone. These involved the effects of the critical interpolated tones. First, it had previously been shown that when the standard and comparison tones are identical in pitch, inclusion in the intervening sequence of a tone that is a semitone higher or lower produces an increase in errors (Deutsch, 1973a). It was therefore reasoned that if in the present experiment $S$ s were sometimes judging the wrong tone, errors might also be made to increase in sequences where the accompanying tones are identical, by including among the interpolated tones a tone that is a semitone higher or lower than this accompanying tone. However, as described in the results section, no effect of such an interpolated tone was found (Conditions $\mathrm{C}$ vs. $\mathrm{A}$ and $\mathrm{B}$ ).

Second, it was reasoned that if $S$ s were at times basing their different judgments on the perceived newness of the lower tone pitch, this might to some extent be counteracted by including a tone of this pitch among the interpolated tones (Deutsch, 1972a). And here a significant effect of the interpolated tone was indeed found, as described in the results section (Conditions D, F vs. E, G). However, this effect was present only in sequences where the lower tone shifted downward but not upward; so the explanation for this phenomenon is unclear. It does, however, provide some indication that judgments of the lower tone pitches were involved. But it must again be stressed that this line of argument can apply only to sequences where the standard and comparison tones were identical in pitch. In sequences where they differed, judgment of the wrong tone would have led to an error pattern opposite to the pattern obtained here.
In sequences where the standard and comparison tones differ in pitch, a substantial increase in errors is produced by including in the interpolated sequence tones of the same pitches as components of the comparison combination. This is as expected from previous studies, and a theoretical basis for this effect has been proposed (Deutsch, 1972a). In the present experiment, this source of confusion is shown to cumulate with the confusion produced by the identical relational context, so that an extremely powerful misrecognition effect is produced. As is shown on Table 3, the error rate changes from $4 \%$ when neither source of confusion is present to $48 \%$ when both sources of confusion occur in the same sequence!

Finally, another hypothesis might be advanced to explain the decrease in pitch comparison performance, here interpreted as due to the presence of conflicting relational information. It will be noted that both in sequences where the standard and comparison tones are identical in pitch and also where they differ, errors are more numerous when the lower tones differ than when they are identical. One might therefore argue that this change in the lower tone pitch produces a general change in the stimulus situation, resulting in a performance decrement that is independent of the storage of tonal relationships. If this were so, then an alteration in the lower tone pitch should produce an increase in errors, irrespective of whether or not the relationship within the combination is preserved by this maneuver. Thus, in sequences where the standard and comparison tones differ, increased errors should also occur when the lower tone shifts in the direction opposite to the direction of difference between the standard and comparison tones. Experi- 
TABLE 3

Effect of Including in Intickpolated Sequence Tones of the Same Pitch as Components of the Comparison Combination (Standard and Comparison 'Tones Different in Pitch)

\begin{tabular}{|c|c|c|c|c|}
\hline \multirow{2}{*}{ Intervals and tones } & \multicolumn{4}{|c|}{ Condition } \\
\hline & H. J & I. K & L, N & $\mathrm{M}, \mathrm{O}$ \\
\hline $\begin{array}{l}\text { Size of interval formed by comparison } \\
\text { combination compared to that formed by } \\
\text { standard combination } \\
\text { Pitch of components of comparison combination } \\
\text { interpolated }\end{array}$ & Different & $\begin{array}{l}\text { Different } \\
\text { Same as com- } \\
\text { parison } \\
\text { tone }\end{array}$ & Same & $\begin{array}{l}\text { Same } \\
\text { Same as both } \\
\text { components } \\
\text { of comparison } \\
\text { combination }\end{array}$ \\
\hline$\%$ errors & 4.4 & 30.7 & 12.5 & 48.4 \\
\hline
\end{tabular}

ment II was designed to control for this possibility.

\section{EXPERIMENT II}

Experiment II was identical to Experiment I, except for the following difference. In the conditions where the standard and comparison tones differed in pitch, whenever their accompanying tones also differed, this was in the opposite direction rather than in parallel. That is, when the comparison tone was a semitone higher than the standard tone, the lower tone shifted downward by a semitone. When the comparison tone was a semitone lower, the lower tone shifted upward. Since the standard combination always formed an interval of seven semitones (a musical fifth) this means that whenever the comparison tone was higher than the standard tone, the comparison combination formed an interval of nine semitones (a musical sixth); and when the comparison tone was lower than the standard tone, the comparison combination formed an interval of five semitones (a musical fourth).

This pattern of relationships has very interesting implications. In music theory, given an interval of $n$ semitones, an interval of $12-n$ semitones is considered equivalent under certain circumstances. The operation relating two such intervals is known in musical terminology as inversion. Now a fourth is a musical inversion of a fifth; however, there is no operation of equivalence linking a fifth to a sixth. Therefore, if our present hypothesis is correct, an increased tendency to misrecognize the comparison tone as identical to the standard tone might also result when the comparison combination forms a fourth, but not when it forms a sixth.

\section{Method}

Conditions. The conditions in Expcriment II were identical to those in Experiment I, except for the following. In Conditions $\mathrm{L}, \mathrm{M}, \mathrm{N}$, and $\mathrm{O}$, the tones accompanying the standard and comparison tones still differed in pitch by a semitone, but now the direction of this shift was opposite to the direction of shift between the standard and comparison tones. These new patterns of relationship are shown on Figure 2. Thus, in Conditions $L$ and $M$, the comparison tone was higher than the standard tone, and the accompanying tone shifted downward; so that the comparison combination formed an interval of nine semitones. In Conditions $\mathrm{N}$ and $\mathrm{O}$, the comparison tone was a semitone lower than the standard tone, and the accompanying tone shifted upward; so that the comparison combination formed an interval of five semitones. As in Experiment I, in Conditions $\mathrm{M}$ and $\mathrm{O}$ a tone of the same pitch as the comparison tone was included in the second serial position of the intervening sequence, and a tone of the same pitch as the tone accompanying the comparison tone was included in the third serial position.

In all other respects the conditions were the same as in Experiment I. The $S$ s listened to the entire tape for 3 separate days, and their results were averaged.

Tonal stimuli. These were the same as those used in Experiment I, except as specified by the experimental conditions.

Apparatus. This was the same as was used in Experiment 1 .

Subjects. Fifteen undergraduates at the University of California at San Diego served as $S$ s for this experiment. They were selected according to the same criteria as for Experiment I and were paid for their services. 
TABLE 4

Effect of Changing Relatronal Context in Experiment II: Standard and Comparison Tones Different in Pitch

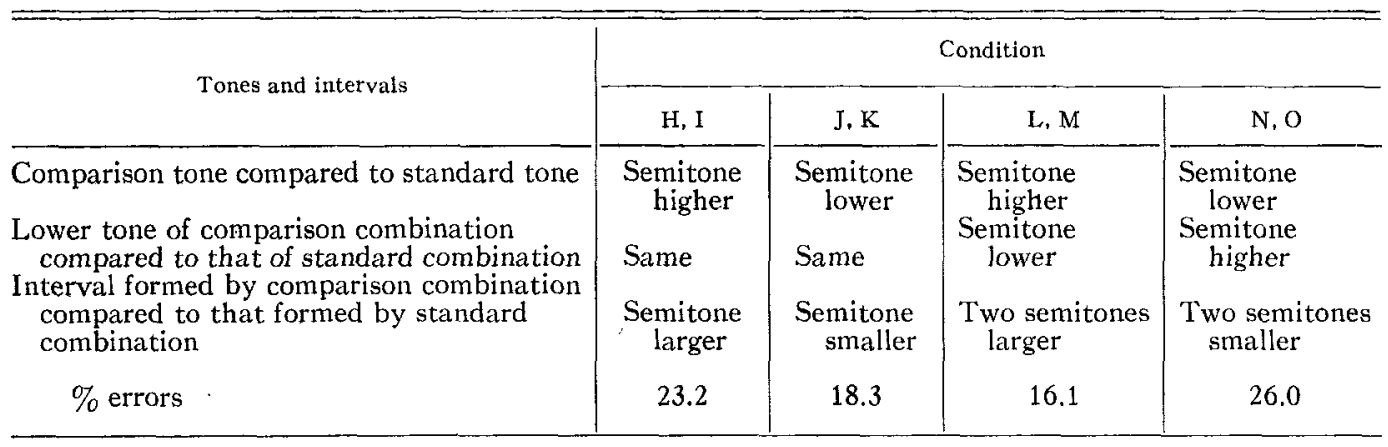

Note. In Conditions $\mathrm{N}, \mathrm{O}$ the interval formed by the comparison combination is an exact musical inversion of the interval formed by the standard combination.

\section{Results}

The results were analyzed in the same fashion as in Experiment I.

Standard and comparison tones identical. The error rates for Conditions A, B, C, D, $\mathrm{E}, \mathrm{F}$, and $\mathrm{G}$ were, respectively, $11.9,10.4$, $8.3,31.1,29.3,36.3$, and 20.4. A $2 \times 2 \times 2$ nested analysis of variance with repeated measures produced the same pattern of errors as in Experiment I. The effect of the lower tone shift was highly significant, $F$ $(1,14)=36.68, p<.01$, as was the effect of the critical intervening tone given a lower tone shift, $F(1,14)=13.58, p<.01$. A significant interaction also occurred between the effect of the critical intervening tone and the direction of lower tone shift, $F(1,14)=6.13, p<.05$. No other significances were obtained.

Standard and comparison tones different. The error rates for Conditions $\mathrm{H}, \mathrm{I}, \mathrm{J}, \mathrm{K}$, $\mathrm{L}, \mathrm{M}, \mathrm{N}$, and $\mathrm{O}$ were, respectively, 10.7, $35.6,6.3,30.4,3.7,28.5,11.9$, and 39.3. Table 4 shows the error rates when the tones were placed in different relational contexts. A $2 \times 2 \times 2$ nested analysis of variance with repeated measures showed the effects of the critical interpolated tones to be highly significant, both when the lower tone shifted, $F(1,28)=42.87, p<$ .01 , and also when it remained the same, $F(1,28)=37.57, p<.01$. However, in contrast to the findings of Experiment I, there was no overall effect of shifting the lower tone pitch, $F<1$. Thus the increase in errors obtained in Experiment I when the lower tone shifted could not have been due to a general change in the stimulus situation, but rather to the preservation of the relationship within the combination. There was also no significant effect of whether the comparison tone was lower or higher than the standard tone in sequences where the lower tone remained the same, $F(1,28)=1.66, p>.05$. However, there was a significant effect where the lower tone also shifted so that the interval formed by the comparison combination was an inversion of the interval formed by the standard combination, $F(1,28)=$ $6.35, p<.05$. There was no interaction between this effect and the effect of the critical interpolated tones, $F<1$.
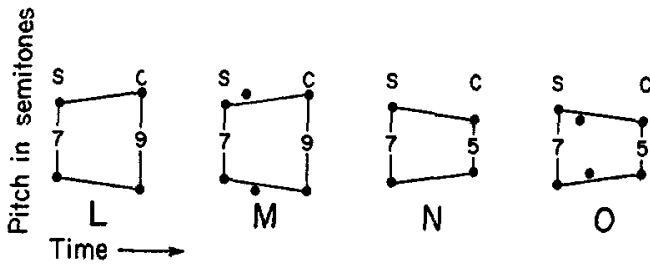

Figure 2. Diagrammatic representation of Conditions L, M, N, and $\mathrm{O}$ of Experiment II. $(\mathrm{S}=$ standard combination, $\mathrm{C}=$ comparison combination. Vertical distance between two circles represents interval size in semitones. Circles placed intermediate between the standard and comparison combinations represent critical intervening tones and their pitches relative to those of the standard and comparison combinations.) 


\section{General Discussion}

Combining the results of both experiments, it can be concluded that when two tones that differ in pitch are placed in an equivalent relational context, there results a tendency for the pitch of one tone to be misrecognized as the same as the other. Similarly, when two tones that are identical in pitch are placed in different relational contexts, there results a tendency for their pitches to be judged as different. Since in these experiments the two components of the standard and comparison combinations were presented to separate ears, these effects could not have been due to patterns of harmonics in the stimulus combinations (Helmholtz, 1862/1954). One question that arises involves the possible mediation of verbal labeling in the confusions generated by the interval information. Accordingly, $S$ s were asked after the experiment to label the intervals they had heard. None of them were able to do this, and upon questioning none were even found to be aware that the difficulty of their task might have been related to the pattern of intervals formed by the standard and comparison combinations. An explanation in terms of verbal mediation therefore appears quite improbable.

The pattern of errors found in Experiment II also sheds light on the organization of memory for tonal relationships. The findings strongly indicate that the storage of interval information involves a shape recognition operation which treats inverted intervals as equivalent. This is in accordance with the findings of Plomp, Wagenaar, and Mimpen (1973), who studied patterns of confusion in judgments of interval by musically trained $S$ s. The intervals employed in their experiment varied in semitone steps from a minor second to an octave. These authors found that $S \mathrm{~s}$ in general confused intervals on the basis of their size; however, they also tended to confuse fifths and fourths with each other more than either with diminished fifths. Confusions between seconds and sevenths and between thirds and sixths also emerged. However, it might be argued that since the $S$ s employed by Plomp et al. were musically sophisticated, their results could have been due to the patterns of equivalences acquired verbally in the course of musical training. Yet, as discussed above, verbal mediation is very unlikely to have played a role in the present experiment.

\section{REFERENCES}

Attneave, F., \& Olson, R. K. Pitch as a medium: A new approach to psychophysical scaling. American Journal of Psychology, 1971, 84, 147-165.

Deutsch, D. Music recognition. Psychological Review, 1969, 76, 300-307.

Deutsch, D. Effect of repetition of standard and comparison tones on recognition memory for pitch. Journal of Experimental Psychology, 1972, 93, 156-162. (a)

Deutsch, D. Mapping of interactions in the pitch memory store. Science, 1972, 175, 1020-1022. (b)

Deutsch, D. Interference in memory between tones adjacent in the musical scale. Journal of Experimental Psychology, 1973, 100, 228-231. (a)

Deutsch, D. Octave generalization of specific interference effects in memory for tonal pitch. Perception \&० Psychophysics, 1973, 13, 271-275. (b)

Helmholtz, H. L. F. On the sensations of tone, as a physiological basis of music. New York: Dover, 1954. (Originally published, 1862.)

Plomp, R., Wagenaar, W. A., \& Mimpen, A. M. $M$ usical interval recognition with simultaneous tones. Acustica, 1973, 29, 102-109.

Wickelgren, W. A. Consolidation and retroactive interference in short-term recognition memory for pitch. Journal of Experimental Psychology, 1966, 72, 250-259.

(Received July 3, 1974) 